\title{
Evolved stars and the origin of abundance trends in planet hosts ${ }^{\star} \star \star$
}

\author{
J. Maldonado ${ }^{1}$ and E. Villaver ${ }^{2}$ \\ 1 INAF-Osservatorio Astronomico di Palermo, Piazza Parlamento 1, 90134 Palermo, Italy \\ e-mail: jmaldonado@astropa.inaf.it \\ 2 Universidad Autónoma de Madrid, Dpto. Física Teórica, Módulo 15, Facultad de Ciencias, Campus de Cantoblanco, 28049 Madrid, \\ Spain
}

Received 3 December 2015 / Accepted 1 February 2016

\begin{abstract}
Context. Detailed chemical abundance studies have revealed different trends between samples of planet and non-planet hosts. Whether these trends are related to the presence of planets or not is strongly debated. At the same time, tentative evidence that the properties of evolved stars with planets may be different from what we know for main-sequence hosts has recently been reported.

Aims. We aim to test whether evolved stars with planets show any chemical peculiarity that could be related to the planet formation process.

Methods. In a consistent way, we determine the metallicity and individual abundances of a large sample of evolved (subgiants and red giants) and main-sequence stars that are with and without known planetary companions, and discuss their metallicity distribution and trends. Our methodology is based on the analysis of high-resolution échelle spectra $(R \gtrsim 57000)$ from $2-3 \mathrm{~m}$ class telescopes. It includes the calculation of the fundamental stellar parameters, as well as individual abundances of $\mathrm{C}, \mathrm{O}, \mathrm{Na}, \mathrm{Mg}, \mathrm{Al}, \mathrm{Si}, \mathrm{S}, \mathrm{Ca}, \mathrm{Sc}$, $\mathrm{Ti}, \mathrm{V}, \mathrm{Cr}, \mathrm{Mn}, \mathrm{Co}, \mathrm{Ni}$, and $\mathrm{Zn}$.

Results. No differences in the $\langle[\mathrm{X} / \mathrm{Fe}]\rangle$ vs. condensation temperature $\left(T_{\mathrm{C}}\right)$ slopes are found between the samples of planet and nonplanet hosts when all elements are considered. However, if the analysis is restricted to only refractory elements, differences in the $T_{\mathrm{C}}$-slopes between stars with and without known planets are found. This result is found to be dependent on the stellar evolutionary stage, as it holds for main-sequence and subgiant stars, while there seems to be no difference between planet and non-planet hosts among the sample of giants. A search for correlations between the $T_{\mathrm{C}}$-slope and the stellar properties reveals significant correlations with the stellar mass and the stellar age. The data also suggest that differences in terms of mass and age between main-sequence planet and non-planet hosts may be present.

Conclusions. Our results are well explained by radial mixing in the Galaxy. The sample of giants contains stars that are more massive and younger than their main-sequence counterparts. This leads to a sample of stars that are possibly less contaminated by stars that were not born in the solar neighbourhood, leading to no chemical differences between planet and non-planet hosts. The sample of main-sequence stars may contain more stars from the outer disc (specially the non-planet host sample) which might lead to the differences observed in the chemical trends.
\end{abstract}

Key words. techniques: spectroscopic - stars: abundances - stars: late-type - planetary systems

\section{Introduction}

Detailed chemical analysis of large samples of stars hosting planets have been revealed as a powerful technique to help us understand how planetary systems form and evolve. However, besides the increasing number of recent studies (e.g. Biazzo et al. 2015; da Silva et al. 2015; Maldonado et al. 2015; Nissen 2015; Ramírez et al. 2015; Thiabaud et al. 2015), the only well established correlation found, so far, is the one that relates the stellar

* Based on observations made with the Mercator Telescope; on observations made with the Nordic Optical Telescope; on observations made with the Italian Telescopio Nazionale Galileo; on observations collected at the Centro Astronómico Hispano Alemán (CAHA) at Calar Alto; and on data products from observations made with ESO Telescopes at the La Silla Paranal Observatory under programme ID 072.C-0488(E), 080.D-0347(A), 081.D-0870(A), 087.C-0831(A), and 183.C-0972(A).

$\star \star$ Tables B.1-B.3 are only available at the CDS via anonymous ftp to cdsarc.u-strasbg. fr $(130.79 .128 .5)$ or via

http://cdsarc.u-strasbg.fr/viz-bin/qcat?J/A+A/588/A98 metallicity with the probability of hosting a gas giant planet (e.g. Gonzalez 1997; Santos et al. 2004; Fischer \& Valenti 2005). Any other claim of a chemical trend in planet-hosting stars has so far been disputed.

Meléndez et al. (2009) report a deficit of refractory elements in the Sun with respect to other solar twins, concluding that it is related to the formation of terrestrial planets. Similar chemical patterns are found by Ramírez et al. $(2009,2010)$ and Gonzalez (2011) in other solar twins and analogs. This interpretation has, however, been challenged by other works that point towards the effects of galactic chemical evolution (González Hernández et al. 2010, 2013) or towards an age/inner galactic origin of the planet host stars as the cause of the detected small chemical depletions (Adibekyan et al. 2014). In a recent work, Maldonado et al. (2015, hereafter MA15) report chemical different slopes in the abundance versus elemental condensation-temperature diagram between stars with cool gas-giant planets and nonplanet hosts, also noting the moderate correlations between the abundance-condensation temperature trend and stellar properties, such as age or metallicity. 
While most of the detailed chemical studies carried so far are around main-sequence (MS) stars, little is known about possible chemical trends in evolved stars with planets. For instance, it is still unclear whether giant stars with planets follow the gasgiant planet stellar metallicity correlation (Sadakane et al. 2005; Schuler et al. 2005; Hekker \& Meléndez 2007; Pasquini et al. 2007; Takeda et al. 2008; Ghezzi et al. 2010a). With the wealth of new planetary discoveries in recent years, we re-visit this issue by performing homogeneous observations and analysis of a large sample of 142 evolved stars. In Maldonado et al. (2013, hereafter MA13), we find that, whilst the metallicity distribution of planet-hosting giant stars with stellar masses $M_{\star}>1.5 M_{\odot}$ follows the general trend that has been established for main sequence stars hosting planets, giant planet hosts in the mass domain $M_{\star} \leq 1.5 M_{\odot}$ do not show metal enrichment. Similar results are found by Mortier et al. (2013). However, Jofré et al. (2015) do not find any clear metallicity difference between giant stars as planet hosts and non-hosts for $M_{\star}>1.5 M_{\odot}$. Reffert et al. (2015) explore the planet occurrence rate with stellar metallicity and stellar mass (exploring the mass range 1.0-3.8 $M_{\odot}$ ) in the UCO/Lick survey. The authors perform a distinction between "secure" (15 stars) planet hosts and planet "candidates" (20 stars), based on their available data and found a strong planet-metallicity correlation among the secure planet hosts, and attribute the lack of correlation found on the sample of planet candidates to the fact that the candidate planets are found preferentially among stars with rather small metallicity and mass. The fact that the bulk of their candidate planets is found among their less massive and low-metallicity stars is intriguing, at least.

Furthermore, the detection of planetary companions is hampered by the large levels of stellar jitter in evolved stars, which is introduced by stellar p-mode oscillations, and which may reach $\sim 100 \mathrm{~m} \mathrm{~s}^{-1}$ (Kjeldsen \& Bedding 1995). Niedzielski et al. (2016) show that the minimum detectable planetary mass is an increasing function of the orbital separation and the stellar luminosity, making the detection of close-in, small planets $\left(M_{\mathrm{p}} \sin i<2 M_{\mathrm{J}}\right.$ within $\left.1 \mathrm{au}\right)$ a difficult task when dealing with evolved stars.

In this paper a detailed analysis of the chemical abundances of a large sample of evolved (subgiants and red giants) stars, with and without planets, is presented. We aim to test whether these stars show any chemical peculiarity and to unravel their origin. This work follows the analysis presented in MA13, but now we extend it to studying possible trends between the abundances and the elemental condensation temperature. This paper is organised as follows. Section 2 describes the stellar samples analysed in this work, the spectroscopic data on which the work is based, and its analysis. The distribution of abundances are presented in Sect. 3. The results are discussed in Sect. 4. Our conclusions follow in Sect. 5.

\section{Data and spectroscopic analysis}

\subsection{Stellar sample}

Figure 1 shows the Hertzsprung-Russell (HR) diagram of the observed stars. The total number of stars amounts to 341 . They are classified as red giants (blue triangles, giants from now on), subgiants (red squares), and main-sequence stars (green circles). The samples of giant and subgiant stars were built taking the stars listed in MA13 for reference, with additional data for 12 new subgiants. The list of MS stars comes from MA13 and M15 works and have been analysed homogeneously. According to their luminosity class and, taking into account the presence

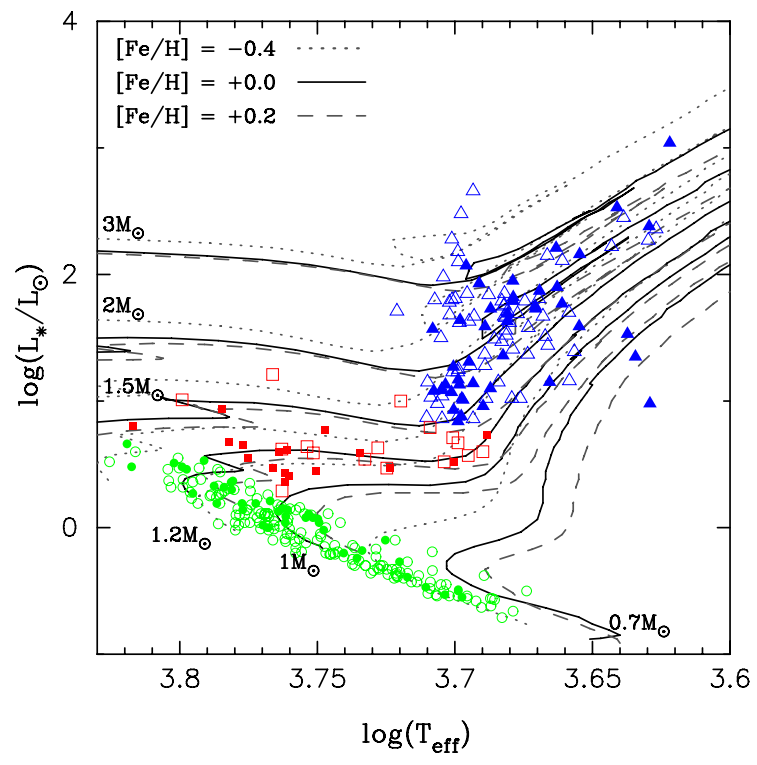

Fig. 1. Luminosity versus $T_{\text {eff }}$ diagram for the observed stars. Giants are plotted with blue triangles, subgiants with red squares, and MS stars with green circles. Filled symbols indicate planet hosts. Some evolutionary tracks, ranging from 0.7 to 3.0 solar masses from Girardi et al. (2000), are overplotted. For each mass, three tracks are plotted, corresponding to $Z=0.008$ ([Fe/H] $=-0.4$ dex, dotted lines), $Z=0.019$ $([\mathrm{Fe} / \mathrm{H}]=+0.0 \mathrm{dex}$, solid lines $)$, and $Z=0.030([\mathrm{Fe} / \mathrm{H}]=+0.20 \mathrm{dex}$, dashed lines).

(or absence) of planetary companions ${ }^{1}$, our sample is divided into 43 giant stars with known planets (hereafter GWPs), 67 giant stars without planets (GWOPs), 16 subgiants hosting planets (SGWPs), 17 subgiants without planets (SGWOPs), 41 MS stars harbouring planets (MSWPs), and 157 MS stars without known planets (MSWOPs). We note that the total number of giant stars known to host planets is 68 so our GWP sample is statistically representative, although it does not include the stellar hosts that are non-observable from the northern hemisphere.

Before continuing with the analysis, we first checked for the presence of biases that might influence the results in our sample. Recently, Reffert et al. (2015) suggested that previous samples of GWPs may be contaminated by "candidate" planet detections, mainly around low-metallicity and low-mass stars, since this seems to be the case in the sample they analysed. To test whether this could be the case, we checked whether our original sample of stars with planets overlap with the list of planets included in the web page that is maintained by these authors ${ }^{2}$. We note that these are radial velocity planets, which have been published in the literature, and therefore each data set is subject to different selection criteria to those that were applied to differentiate secure/candidate planets in the sample of Reffert et al. (2015). Obviously, the criteria used by those authors - to include (or not) a planet detection on their web-page - may be discussed, but this is beyond the scope of this work. Here, we just assumed their criteria is valid and check for biases in our sample. From our original list of 43 GWPs, 27 overlap with the aforementioned list (hereafter RL planet hosts), while 16 are not included in this list (nRL planet hosts). The analysis of the metallicity distribution of RL/nRL planet hosts, Fig. 2 (left), reveals

\footnotetext{
1 According to the available data at the Extrasolar Planets Encyclopaedia, http://exoplanet.eu/

2 http://www. Isw. uni-heidelberg.de/users/sreffert/ giantplanets.html
} 

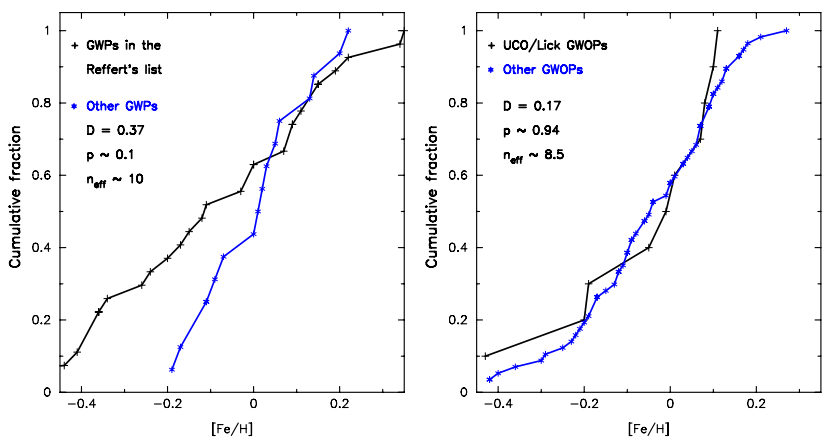

Fig. 2. Cumulative $[\mathrm{Fe} / \mathrm{H}]$ distribution for giant stars. Left: GWPs, divided into planet hosts included in the Reffert's list (27 stars) and planet hosts not included in this list (16 stars). Right: GWOPs, divided into stars included in the UCO/Lick survey (10 stars) and GWOPs not included in this survey (57 stars). See text for details.

that the nRL planet hosts do not show lower metallicities, but actually higher metallicities than the RL planet hosts. While the median metallicity of the RL planet hosts is -0.11 dex, the $n R L$ planet hosts show a median value of +0.02 dex. Furthermore, a two-sided Kolmogorov-Smirnov (KS) test returns a probability of $\sim 0.1$ of both distributions being drawn from the same parent population. Therefore, we conclude that the metallicity distribution of the 16 planets, which were not included in the Reffert's list, do not seem to contaminate our sample towards low-metallicity stars.

Our GWOP sample was selected, based on available giant stars from the Massarotti et al. (2008) list of HIPPARCos giants within $100 \mathrm{pc}$ from the Sun. So, in principle, we cannot rule out the possibility that some of these stars host an undetected gas-giant planet. Reffert et al. (2015) claim a planet detection of $4-5 \%$ in their sample. A slightly higher detection rate of $10-15 \%$ is provided by the Thüringer Landessternwarte survey (Döllinger et al. 2009). Johnson et al. (2011) analyse 246 subgiants from the California Planet Search, providing a detection rate of $15 \%$. These numbers are consistent with the models by Kennedy \& Kenyon (2008), which predict a frequency of gasgiant planets of $10 \%$ around $1.5 M_{\odot}$ stars. To test whether our GWOP sample might be contaminated, we divided it into two subsamples: one including those stars, which have been monitored in the UCO/Lick survey, as listed in Reffert et al. (2015), and which have not been reported as having a detected planet (10 stars); and another subsample, without our GWOP stars included in this survey (57 stars). Figure 2 (right) shows the metallicity distribution of both subsamples. From the plot, it is clear that they are almost identical. A KS test provides a probability of $94 \%$ of both subsamples having similar metallicity distributions. Thus, we conclude that it is very unlikely that the properties of our GWOP (such as metallicity or elemental abundances) are affected in a significant way by the presence of undetected gas-giant planets. This result seems in line with the contamination expected in the GWOP sample (as seen, at most at the $10 \%$ level), which is too small to significantly affect the results/shift the metallicity distributions.

With regards less massive planets $\left(M_{\mathrm{p}} \sin i<30 M_{\oplus}\right)$, there is increasing evidence that they might be common around MS solar-type stars (e.g. Mayor et al. 2011; Cassan et al. 2012; Howard et al. 2013), which may certainly contaminate the MSWOP sample. We note that stars hosting low-mass planets do not seem to be preferentially metal-rich (Ghezzi et al. 2010b; Mayor et al. 2011; Sousa et al. 2011; Buchhave et al. 2015; although see Buchhave \& Latham 2015; for an opposing view Wang \& Fischer 2015). Furthermore, MA15 find that stars with low-mass planets show similar chemical trends to stars without known planetary companions. Therefore we discarded those stars that were harbouring low-mass planets from our MSWP sample.

Regarding giant stars, the detection of low-mass planets is hindered around this kind of star (see e.g. Niedzielski et al. 2016). Therefore any hint of giant stars hosting more massive planets than MS stars (e.g. MA13) needs to be interpreted with caution as the larger levels of stellar oscillation in evolved stars certainly introduces an observational bias against the detection of low-mass planets via the radial-velocity method. In addition, it is relevant to note that low-mass planets have more chances of surviving the processes that take place when an MS star evolves off the MS since there is a strong dependence of the tidal forces on the mass ratio of the planet-star system (e.g. Villaver \& Livio 2009; Mustill \& Villaver 2012; Villaver et al. 2014).

We finally note that the mass distribution of our giant stars is similar to those of more general exoplanet search projects, which were focused on evolved stars (see next subsection for details on this calculation). Our evolved stars sample covers a mass range between 1.0 and 3.6 $M_{\odot}$, having a peak at $\sim 1.5 M_{\odot}$, and then decreases steadily, as does the mass distribution of the giant stars of the Pennsylvania-Torun Planet Search survey (PTPS) or the Retired A Stars project (see Niedzielski et al. 2016, Fig. 13c).

Recent works have put into question the reliability of the masses of evolved stars that host planetary systems (Lloyd 2011, 2013; Schlaufman \& Winn 2013). Therefore, a comparison between our derived masses and those given in the PTPS (Zieliński et al. 2012; Niedzielski et al. 2016) and in the UCO/Lick (Reffert et al. 2015) surveys was performed. The masses provided in other surveys may have been overestimated (see Niedzielski et al. 2016) and thus we do not use them for comparison with our sample. The Retired A stars and their Companions survey (Ghezzi \& Johnson 2015) also provide masses that are consistent with the PTPS and Lick surveys, however, we do not have enough stars in common to include them in the analysis. The comparison is shown in Fig. 3. It reveals an overall good agreement between our mass estimates and those by PTPS and Lick surveys. We note that the median difference is only $0.03,0.04$ solar masses with an rms standard deviation of 0.23 , and $0.24 M_{\odot}$ for the PTPS and Lick surveys respectively.

\subsection{Spectroscopic analysis}

The spectroscopic data used in this work is basically the same as used in MA13 and MA15 to which we refer to for further details. In brief, high-resolution spectra of the stars were obtained: i) at La Palma observatory (Canary Islands, Spain) with the HERMES spectrograph (Raskin et al. 2011) at the Mercator telescope; ii) at the Nordic Optical Telescope with the FIES (Frandsen \& Lindberg 1999) instrument; iii) at the 2.2-m telescope of the Calar Alto observatory (CAHA, Almería, Spain) using the FOCES (Pfeiffer et al. 1998) spectrograph; iv) at the Telescopio Nazionale Galileo (TNG, $3.58 \mathrm{~m}$ ) using the SARG (Gratton et al. 2001) spectrograph. Additional spectra from the public library " $\mathrm{S}^{4} \mathrm{~N}$ " (Allende Prieto et al. 2004) as well as HARPS and FEROS spectra from the ESO Archive Facility ${ }^{3}$ were also used.

We are aware that, ideally, all our targets should have been observed with the same spectrograph and configuration. However, all the spectra used in this work have high-resolution

http://archive.eso.org/wdb/wdb/adp/phase3_main/form 


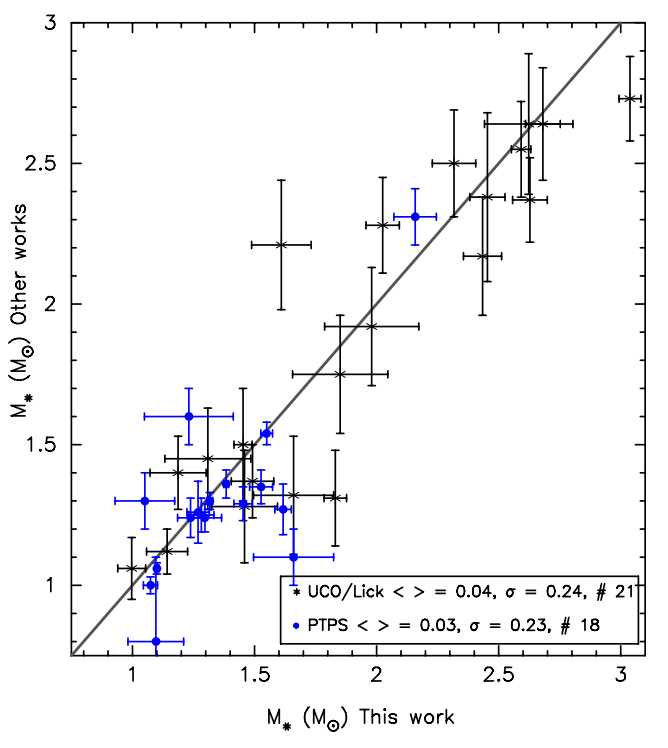

Fig. 3. Stellar mass values estimates from the literature versus the values obtained in this work. The symbol \langle\rangle in the legend represents the median difference. The grey continuous line represents the 1:1 relation.

(from 42 000 of FEROS spectra to 115000 for HARPS), have a high signal-to-noise ratio (median value 107 at $6050 \AA$ ) and cover a wide spectral range (from 3780-6910 $\AA$ for HARPS to 3400-10900 ̊̊ for McDonald) with enough lines for a highquality abundance determination.

Basic stellar parameters $T_{\text {eff }}, \log g$, microturbulent velocity $\xi_{\mathrm{t}}$, and $[\mathrm{Fe} / \mathrm{H}]$ are determined using the code $\mathrm{TGVIT}^{4}$ (Takeda et al. 2005), which implements the iron ionisation and excitation equilibrium conditions, and which is a methodology that is widely applied to solar-like stars. The line list, as well as the adopted parameters (excitation potential, $\log (g f)$ values) can be found on Y. Takeda's web page. This code makes use of ATLAS9, plane-parallel, LTE atmosphere models (Kurucz 1993).

Chemical abundance of individual elements $\mathrm{C}, \mathrm{O}, \mathrm{Na}, \mathrm{Mg}$, $\mathrm{Al}, \mathrm{Si}, \mathrm{S}, \mathrm{Ca}, \mathrm{Sc}, \mathrm{Ti}, \mathrm{V}, \mathrm{Cr}, \mathrm{Mn}, \mathrm{Co}, \mathrm{Ni}$, and $\mathrm{Zn}$ were obtained

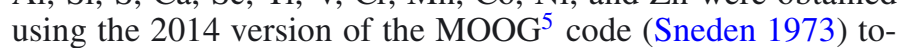
gether with ATLAS9 atmosphere models (Kurucz 1993). The measured equivalent widths (EWs) of a list of narrow, nonblended lines for each of the aforementioned species are used as inputs. The selected lines are taken from the list provided by MA15. Hyperfine structure (HFS) was taken into account for V I and Co I abundances. HFS corrections for Mn I were not taken into account since, in MA15, we found slightly different abundances when considering different lines. Although HFS effects may be present for other elements (e.g. Mg I, Sc I), we do not expect these effects to bias the results of the comparisons, which were performed in this work, between samples of stars with and without planets, given that they have otherwise similar properties.

The oxygen abundance was derived from the forbidden [O I] line at $6300 \AA$. This line is well known for being blended with a closer Ni I line (e.g. Allende Prieto et al. 2001). We made use of the MOOG driver ewfind to determine the EW of the Ni line using the previously derived Ni abundance. This EW was subtracted from the measured EW of the of the Ni I

\footnotetext{
4 http://optik2.mtk.nao.ac.jp takeda/tgv/

5 http://www.as.utexas.edu/ chris/moog.html
}

plus [ $\left[\begin{array}{ll}\mathrm{O} & \mathrm{I}\end{array}\right]$ feature. Then, the oxygen abundance was determined from the remaining EW (e.g. Delgado Mena et al. 2010; González Hernández et al. 2013). Since oxygen abundances in MA15 stars were derived from the O I triplet lines at $777 \mathrm{~nm}$, we have recomputed them using the [O I] $630 \mathrm{~nm}$ line. The lines at 505.2 and $538.0 \mathrm{~nm}$ were used instead of those reported in MA15 for the carbon abundance, since we found the MA15 lines gave abnormally high abundances for the giant stars. Several problems have been reported by da Silva et al. (2011) when using the $505.2 \mathrm{~nm}$ line. However, we do not find significant differences between the abundances derived from the $505.2 \mathrm{~nm}$ line and the ones derived from the $538.0 \mathrm{~nm}$ line, being the mean and median differences around $\sim 0.02$.

Evolutionary parameters, namely stellar mass, radius, and age, were computed using the PARAM ${ }^{6}$ code (da Silva et al. 2006) with the new PARSEC isochrones from Bressan et al. (2012).

Our derived stellar parameters are given in Table B.1, whilst the abundances are provided in Table B.2. The recomputed abundances of carbon and oxygen for those stars taken from MA15 are given in Table B.3. These tables are available at the CDS.

\section{Analysis}

\section{1. $[\mathrm{X} / \mathrm{Fe}]-T_{\mathrm{C}}$ trends}

Chemical differences were searched for by studying possible trends between the abundances, $[\mathrm{X} / \mathrm{Fe}]$, and the elemental condensation temperature, $T_{\mathrm{C}}$. Mean abundances for each of the samples were computed, and the $T_{\mathrm{C}}$-slope was derived by performing a linear fit, weighting each element by its corresponding star-to-star scatter. Values of $T_{\mathrm{C}}$ correspond to a $50 \%$ equilibrium condensation temperature for a solar system composition gas (Lodders 2003).

As in MA15, we compute the slope of the $[\mathrm{X} / \mathrm{Fe}]$ vs. $T_{\mathrm{C}}$ fit considering, firstly, all refractory and volatile elements $\left(T_{\mathrm{C}}^{\text {all }}-\right.$ slope), and then considering only refractories ( $T_{\mathrm{C}}^{\mathrm{refrac}}$-slope). In this way, we take into account the fact that the abundances of volatiles are, in general, more difficult to obtain accurately ${ }^{7}$. A Monte Carlo simulation was carried out to give a significance for the derived slopes. We created $10^{4}$ series of simulated random abundances and errors, keeping the media and the standard deviation of the original data. For each series of simulated data the corresponding $T_{\mathrm{C}}$-slope was derived. Assuming that the distribution of the simulated slopes follows a Gaussian function, we then compute the probability that the simulated slope takes the value found when fitting the original data (hereafter $p$-value). The corresponding plots are shown in Fig. 4, and a summary of the fits is presented in Table 1.

Left panel in Fig. 4 shows that when all elements are considered there seems to be no difference in the chemical behaviour of the planet host samples in relation to their respective comparison samples. This result holds independently of the evolutionary state of the stars (giant, subgiant or main-sequence), having stars with and without planets very similar slopes. We note, however, that the slopes of MS and subgiant stars tend, within the errors, to be consistent with zero, with moderate $p$-values. Giants, on the other hand, show clearly negative slopes and statistically significant low $p$-values.

\footnotetext{
6 http://stev.oapd.inaf.it/cgi-bin/param

7 We consider those elements with $T_{\mathrm{C}}$ lower than $900 \mathrm{~K}$ as being volatile, namely $\mathrm{C}, \mathrm{O}, \mathrm{S}$, and $\mathrm{Zn}$.
} 
J. Maldonado and E. Villaver: Evolved stars and the origin of abundance trends in planet hosts
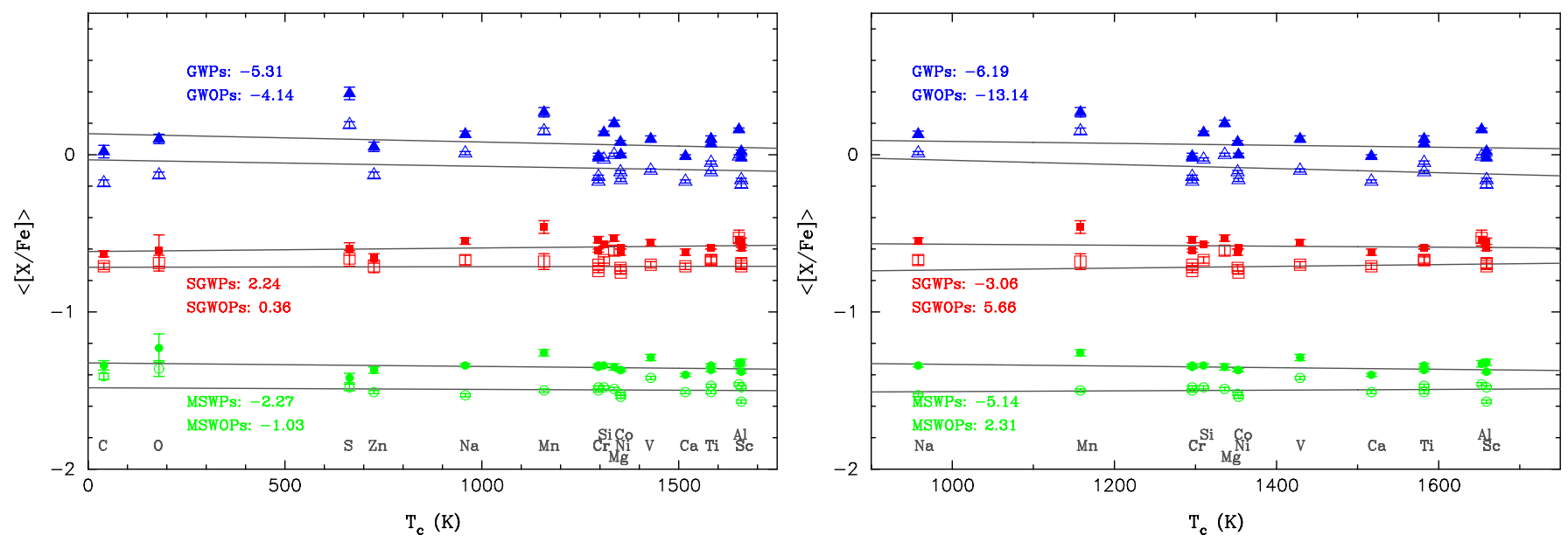

Fig. 4. $\langle[\mathrm{X} / \mathrm{Fe}]\rangle-T_{\mathrm{C}}$ trends of all the stars analysed. Giants are plotted with blue triangles, subgiants with red squares, and MS stars with green circles. Filled symbols indicate planet hosts. Each planet host subsample is shown against its corresponding comparison subsample (e.g. GWPs vs. GWOPs) with an offset of -0.15 for the sake of clarity. The offset between giants, subgiants, and MS samples is -0.75 . For guidance, the derived slopes are shown in the plots (units of $10^{-5} \mathrm{dex} / \mathrm{K}$ ). The left panel shows the $\langle[\mathrm{X} / \mathrm{Fe}]\rangle-T_{\mathrm{C}}$ trends when all elements (volatiles and refractories) are taken into account, whilst the right one shows the $\langle[\mathrm{X} / \mathrm{Fe}]\rangle-T_{\mathrm{C}}$ trends when only refractories are considered.

Table 1. Results of the $\langle[\mathrm{X} / \mathrm{Fe}]\rangle-T_{\mathrm{C}}$ linear fits.

\begin{tabular}{lcccc}
\hline \hline Sample & $\begin{array}{c}\text { All elements } \\
\text { Slope }\left(\times 10^{-5} \mathrm{dex} / \mathrm{K}\right)\end{array}$ & $p$ & $\begin{array}{c}\text { Only refractory } \\
\text { Slope }\left(\times 10^{-5} \mathrm{dex} / \mathrm{K}\right)\end{array}$ & $p$ \\
\hline GWPs & $-5.31 \pm 1.20$ & 0.06 & $-6.19 \pm 1.84$ & 0.07 \\
GWOPs & $-4.14 \pm 0.81$ & 0.07 & $-13.14 \pm 1.45$ & 0.05 \\
\hline SGWPs & $2.24 \pm 1.17$ & 0.12 & $-3.06 \pm 2.32$ & 0.17 \\
SGWOPs & $0.36 \pm 1.29$ & 0.17 & $5.66 \pm 3.35$ & 0.17 \\
\hline MSWPs & $-2.27 \pm 1.11$ & 0.11 & $-5.14 \pm 1.53$ & 0.13 \\
MSWOPs & $-1.03 \pm 0.79$ & 0.08 & $2.31 \pm 1.32$ & 0.19 \\
\hline
\end{tabular}

Notes. For each fit, its probability of slope being by chance $(p)$ is also given.

The $[\mathrm{X} / \mathrm{Fe}]$ vs. $T_{\mathrm{C}}$ trends, when only the refractory elements are considered, are shown in the right panel of Fig. 4. We note a slight change in behaviour between MSWPs (negative slope) with respect to MSWOPs (positive slope). This tendency is also seen in the samples of subgiant stars (i.e., negative slopes in planet hosts, positive slopes in non-planet hosts). The statistical significance of these trends is however low, with moderate $p$-values. When considering giant stars, both GWP and GWOP samples show negative $[\mathrm{X} / \mathrm{Fe}]$ vs. $T_{\mathrm{C}}$ trends, with the slope of the GWOP sample being slightly more negative. We note that the slopes obtained for the giant samples are statistically significant but that is not the case for the MS and subgiant samples.

We also note that, at this point, we are considering the sample of giants as a whole, i.e. without any mass differentiation, despite the reported difference in the metallicity behaviour between stars with masses lower and larger than $1.5 M_{\odot}$. We will analyse the mass segregation in detail in Sect. 3.3. We caution that other effects (e.g. uncertainties in the stellar mass determination, or the criteria used to discern subgiants from giants) may also be present.

\subsection{Trends with evolutionary properties}

In the previous subsection we have found that a different chemical trend may exist between planets and non-planets hosts when only refractory elements are considered, but that this difference seem to be only present in MS and subgiant stars, and not in giants. This behaviour resembles the gas-giant planet-metallicity correlation known to hold for MS and subgiant stars, but which is controversial when considered for giants (e.g. MA13). It is, therefore, natural to ask whether the obtained abundance trends correlate with other evolutionary parameters.

We have thus performed a search for correlations between the derived $T_{\mathrm{C}}$-slopes for each individual star and the evolutionary parameters, namely surface gravity, stellar mass, age, and radius. Stellar metallicity and the stellar mean galactocentric distance $\left(d_{\text {galact }}\right)$ have also been considered with values taken from our own previous work and from Casagrande et al. (2011), respectively. Two kinds of analysis have been performed. The first one consists of the classical Spearman's correlation test. Further analysis includes the evaluation of the significance of the correlations by a bootstrap Monte Carlo (MC) test plus a Gaussian random shift of each data-point within its error bars. The tests were done using the MCSPEARMAN ${ }^{8}$ code by Curran (2014), and the results are shown in Table 2. It is clear from this table that the classical analysis suggests moderate but highly significant correlations between the $T_{\mathrm{C}}$-slopes and the evolutionary parameters. The MC simulations do not exclude such dependencies, however they suggest that the correlations are weak, with the $z$-score values in all cases lower than $3 \sigma$.

Our analysis show that the derived $T_{\mathrm{C}}$-slope correlates with the stellar metallicity. This kind of correlation suggests that Galactic chemical evolution (GCE) effects may be having an impact on our derived abundance patterns. While some authors (González Hernández et al. 2013; Adibekyan et al. 2014) have tried to account for these effects by fitting straight lines to the $[\mathrm{X} / \mathrm{Fe}]$ vs. $[\mathrm{Fe} / \mathrm{H}]$ plots, others (e.g. Ramírez et al. 2014) argue that correcting from GCE effects in this manner may prevent us from finding elemental depletions that are due to planet formation.

Table 2 also shows a clear correlation between the $T_{\mathrm{C}}$-slope and the stellar mass, or the stellar age (see the corresponding $p$-values). We note that the correlations were performed using all stars (planet and non-planet hosts) together. Less massive

8 https://github.com/PACurran/MCSpearman/ 
Table 2. Results from the Spearman's correlation test between the $T_{\mathrm{C}}$ slopes and different stellar properties.

\begin{tabular}{|c|c|c|c|c|c|c|c|c|c|}
\hline \multirow[b]{3}{*}{ Parameter } & \multirow[b]{3}{*}{ Sample } & \multicolumn{4}{|c|}{ All elements } & \multicolumn{4}{|c|}{ Only refractory } \\
\hline & & \multicolumn{2}{|c|}{$\mathrm{MC}$} & \multicolumn{2}{|c|}{ CA } & \multicolumn{2}{|c|}{$\mathrm{MC}$} & \multicolumn{2}{|c|}{ CA } \\
\hline & & SR & $\mathrm{ZS}$ & SR & $p$ & SR & $\mathrm{ZS}$ & SR & $p$ \\
\hline$[\mathrm{Fe} / \mathrm{H}]$ & $\begin{array}{l}\text { All } \\
\text { Giants } \\
\text { Subgiants } \\
\text { MS }\end{array}$ & $\begin{array}{l}0.29 \pm 0.05 \\
0.44 \pm 0.09 \\
0.49 \pm 0.15 \\
0.15 \pm 0.07\end{array}$ & $\begin{array}{l}2.29 \pm 0.45 \\
2.10 \pm 0.47 \\
1.30 \pm 0.49 \\
0.92 \pm 0.43\end{array}$ & $\begin{array}{l}0.31 \\
0.48 \\
0.52 \\
0.16\end{array}$ & $\begin{array}{c}\sim 10^{-8} \\
\sim 10^{-7} \\
\sim 10^{-3} \\
0.02\end{array}$ & $\begin{array}{l}-0.32 \pm 0.05 \\
-0.42 \pm 0.09 \\
-0.55 \pm 0.15 \\
-0.28 \pm 0.07\end{array}$ & $\begin{array}{l}-2.57 \pm 0.45 \\
-1.96 \pm 0.45 \\
-1.48 \pm 0.52 \\
-1.69 \pm 0.45\end{array}$ & $\begin{array}{l}-0.37 \\
-0.51 \\
-0.60 \\
-0.32\end{array}$ & $\begin{array}{l}\sim 10^{-12} \\
\sim 10^{-8} \\
\sim 10^{-4} \\
\sim 10^{-6}\end{array}$ \\
\hline $\log g$ & $\begin{array}{l}\text { All } \\
\text { Giants } \\
\text { Subgiants } \\
\text { MS }\end{array}$ & $\begin{array}{c}-0.16 \pm 0.05 \\
0.26 \pm 0.10 \\
0.30 \pm 0.17 \\
-0.17 \pm 0.07\end{array}$ & $\begin{array}{c}-1.27 \pm 0.44 \\
1.17 \pm 0.45 \\
0.75 \pm 0.45 \\
-1.00 \pm 0.41 \\
\end{array}$ & $\begin{array}{c}-0.18 \\
0.28 \\
0.33 \\
-0.19 \\
\end{array}$ & $\begin{array}{c}\sim 10^{-3} \\
\sim 10^{-3} \\
0.06 \\
\sim 0.01 \\
\end{array}$ & $\begin{array}{c}0.22 \pm 0.05 \\
0.17 \pm 0.10 \\
-0.33 \pm 0.17 \\
0.16 \pm 0.07\end{array}$ & $\begin{array}{c}1.75 \pm 0.44 \\
0.74 \pm 0.43 \\
-0.82 \pm 0.45 \\
0.98 \pm 0.44 \\
\end{array}$ & $\begin{array}{c}0.28 \\
0.22 \\
-0.36 \\
0.21 \\
\end{array}$ & $\begin{array}{c}\sim 10^{-7} \\
0.02 \\
0.04 \\
\sim 10^{-3} \\
\end{array}$ \\
\hline$M_{\star}$ & $\begin{array}{l}\text { All } \\
\text { Giants } \\
\text { Subgiants } \\
\text { MS }\end{array}$ & $\begin{array}{l}0.34 \pm 0.05 \\
0.43 \pm 0.08 \\
0.29 \pm 0.17 \\
0.31 \pm 0.07\end{array}$ & $\begin{array}{l}2.70 \pm 0.43 \\
2.00 \pm 0.43 \\
0.71 \pm 0.42 \\
1.87 \pm 0.45\end{array}$ & $\begin{array}{l}0.36 \\
0.50 \\
0.32 \\
0.33 \\
\end{array}$ & $\begin{array}{c}\sim 10^{-11} \\
\sim 10^{-8} \\
0.08 \\
\sim 10^{-6} \\
\end{array}$ & $\begin{array}{l}-0.37 \pm 0.05 \\
-0.57 \pm 0.07 \\
-0.23 \pm 0.19 \\
-0.32 \pm 0.07\end{array}$ & $\begin{array}{l}-2.97 \pm 0.45 \\
-2.84 \pm 0.48 \\
-0.55 \pm 0.47 \\
-1.96 \pm 0.46 \\
\end{array}$ & $\begin{array}{l}-0.45 \\
-0.70 \\
-0.25 \\
-0.39 \\
\end{array}$ & $\begin{array}{c}\sim 10^{-17} \\
\sim 10^{-17} \\
0.17 \\
\sim 10^{-8} \\
\end{array}$ \\
\hline Age & $\begin{array}{l}\text { All } \\
\text { Giants } \\
\text { Subgiants } \\
\text { MS }\end{array}$ & $\begin{array}{l}-0.11 \pm 0.06 \\
-0.35 \pm 0.09 \\
-0.31 \pm 0.16 \\
-0.02 \pm 0.07\end{array}$ & $\begin{array}{l}-0.82 \pm 0.43 \\
-1.62 \pm 0.43 \\
-0.76 \pm 0.42 \\
-0.10 \pm 0.42\end{array}$ & $\begin{array}{l}-0.14 \\
-0.47 \\
-0.35 \\
-0.03 \\
\end{array}$ & $\begin{array}{c}\sim 0.01 \\
\sim 10^{-7} \\
0.05 \\
0.72 \\
\end{array}$ & $\begin{array}{l}0.25 \pm 0.05 \\
0.52 \pm 0.08 \\
0.10 \pm 0.20 \\
0.15 \pm 0.07 \\
\end{array}$ & $\begin{array}{l}1.94 \pm 0.44 \\
2.52 \pm 0.49 \\
0.23 \pm 0.49 \\
0.86 \pm 0.42 \\
\end{array}$ & $\begin{array}{l}0.31 \\
0.65 \\
0.11 \\
0.17 \\
\end{array}$ & $\begin{array}{c}\sim 10^{-8} \\
\sim 10^{-14} \\
0.54 \\
0.02 \\
\end{array}$ \\
\hline$R_{\star}$ & $\begin{array}{l}\text { All } \\
\text { Giants } \\
\text { Subgiants } \\
\text { MS }\end{array}$ & $\begin{array}{c}0.23 \pm 0.05 \\
-0.11 \pm 0.10 \\
-0.19 \pm 0.19 \\
0.29 \pm 0.07 \\
\end{array}$ & $\begin{array}{c}1.79 \pm 0.44 \\
-0.48 \pm 0.45 \\
-0.44 \pm 0.46 \\
1.74 \pm 0.45 \\
\end{array}$ & $\begin{array}{c}0.25 \\
-0.11 \\
-0.22 \\
0.31 \\
\end{array}$ & $\begin{array}{c}\sim 10^{-6} \\
0.27 \\
0.22 \\
\sim 10^{-5} \\
\end{array}$ & $\begin{array}{c}-0.27 \pm 0.05 \\
-0.31 \pm 0.09 \\
0.13 \pm 0.18 \\
-0.24 \pm 0.07\end{array}$ & $\begin{array}{c}-2.09 \pm 0.45 \\
-1.41 \pm 0.43 \\
0.32 \pm 0.43 \\
-1.41 \pm 0.46 \\
\end{array}$ & $\begin{array}{c}-0.33 \\
-0.39 \\
0.14 \\
-0.29 \\
\end{array}$ & $\begin{array}{c}\sim 10^{-9} \\
\sim 10^{-5} \\
0.43 \\
\sim 10^{-5} \\
\end{array}$ \\
\hline$d_{\text {galact }}$ & All & $0.00 \pm 0.08$ & $0.03 \pm 0.44$ & 0.00 & 0.99 & $-0.21 \pm 0.07$ & $-1.20 \pm 0.44$ & -0.22 & $\sim 10^{-3}$ \\
\hline
\end{tabular}

Notes. MC stands for Monte Carlo simulation, CA for "classical” analysis, SR for Spearman's correlation rank coefficient, ZS means $z$-score, and $p$ denotes the significance of the SR coefficient.

and older stars show more positive $T_{\mathrm{C}}^{\mathrm{ref}}$-slopes, and more negative $T_{\mathrm{C}}^{\text {all }}$-slopes. This result agrees with recent studies of solar twins in which the existence of a correlation between $[\mathrm{X} / \mathrm{Fe}]$ and the stellar age have been revealed (Nissen 2015; Spina et al. 2016). Following this line of reasoning, a comparison of the stellar masses and ages between planet hosts and non-planet hosts were performed. Figures 5 and 6 show the corresponding cumulative distribution functions, while some statistical diagnostics are presented in Table 3. These figures show that there seems to be a hint of MS and subgiant non-planet hosts having slightly smaller masses and older ages than planet hosts. This could be a selection effect since radial velocity surveys tend to target stars with low levels of activity. We note that, in the case of giants, the behaviour seems to be the opposite, being GWOPs that are slightly younger and massive than GWPs.

To test the statistical significance of these trends, several KS tests were performed (Table 4). The results from the KS test show that the differences in mass or age between planet and non-planet hosts are in general not significant from an statistical point of view. The mass segregation between planet and nonplanet hosts in MS stars appears to be the only trend that might be statistically significant.

In order to check whether GCE effects my affect or not our results, abundances were corrected by fitting straight lines to the $[\mathrm{X} / \mathrm{Fe}]$ vs. $[\mathrm{Fe} / \mathrm{H}]$ plots (see Fig. A.1). As before, $T_{\mathrm{C}}$-slopes were computed for each individual star and a search for correlations was performed. We find that most of the correlations with the evolutionary parameters remain.

Finally, we have redone our analysis, but only taking into consideration those stars that are similar to our Sun (the so-called solar analogs). The results are given in Table 5. We note that
Table 3. Stellar mass and stellar age statistics of the stellar samples.

\begin{tabular}{lccccc}
\hline \hline \multicolumn{7}{c}{ Stellar mass $\left(M_{\odot}\right)$} \\
\hline Sample & Mean & Median & $\sigma$ & Min. & Max. \\
\hline MSWPs & 1.06 & 1.03 & 0.16 & 0.79 & 1.48 \\
MSWOPs & 0.95 & 0.94 & 0.14 & 0.68 & 1.37 \\
SGWPS & 1.25 & 1.30 & 0.13 & 1.03 & 1.49 \\
SGWOPs & 1.24 & 1.19 & 0.22 & 0.93 & 1.62 \\
GWPs & 1.60 & 1.48 & 0.48 & 1.01 & 3.04 \\
GWOPs & 1.76 & 1.60 & 0.56 & 1.00 & 3.62 \\
\hline \multicolumn{7}{c}{ Stellar age (Gyr) } \\
\hline Sample & Mean & Median & $\sigma$ & Min. & Max. \\
\hline MSWPs & 3.41 & 3.02 & 2.41 & 0.29 & 9.99 \\
MSWOPs & 4.32 & 3.28 & 3.62 & 0.10 & 11.48 \\
SGWPs & 5.00 & 4.32 & 1.98 & 1.01 & 8.63 \\
SGWOPs & 5.62 & 4.76 & 2.88 & 2.01 & 11.48 \\
GWPs & 3.37 & 2.95 & 2.15 & 0.38 & 10.10 \\
GWOPs & 2.82 & 2.29 & 2.15 & 0.24 & 9.28 \\
\hline \multicolumn{7}{c}{}
\end{tabular}

the correlations discussed before are not so evident. However, the classical analysis suggests that some correlations may still be present. In particular between $T_{\mathrm{C}}^{\text {all }}$ and $\operatorname{logg}$, and perhaps the stellar age, and also between $T_{\mathrm{C}}^{\mathrm{ref}}$ and $[\mathrm{Fe} / \mathrm{H}]$. A more detailed, strictly differential analysis of these stars should be done to clarify this point and to properly compare with previous results (e.g. Adibekyan et al. 2014; da Silva et al. 2015). 
J. Maldonado and E. Villaver: Evolved stars and the origin of abundance trends in planet hosts
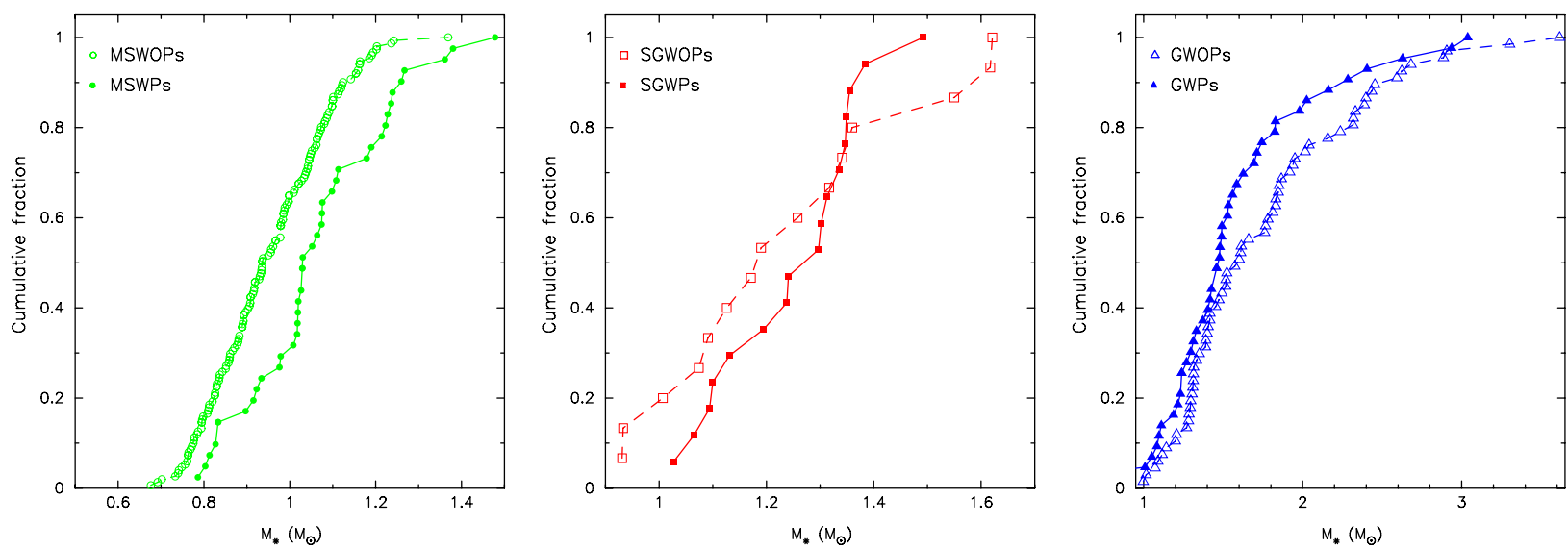

Fig. 5. Cumulative distribution function of stellar masses for MS stars (left), subgiants (middle), and giants (right).
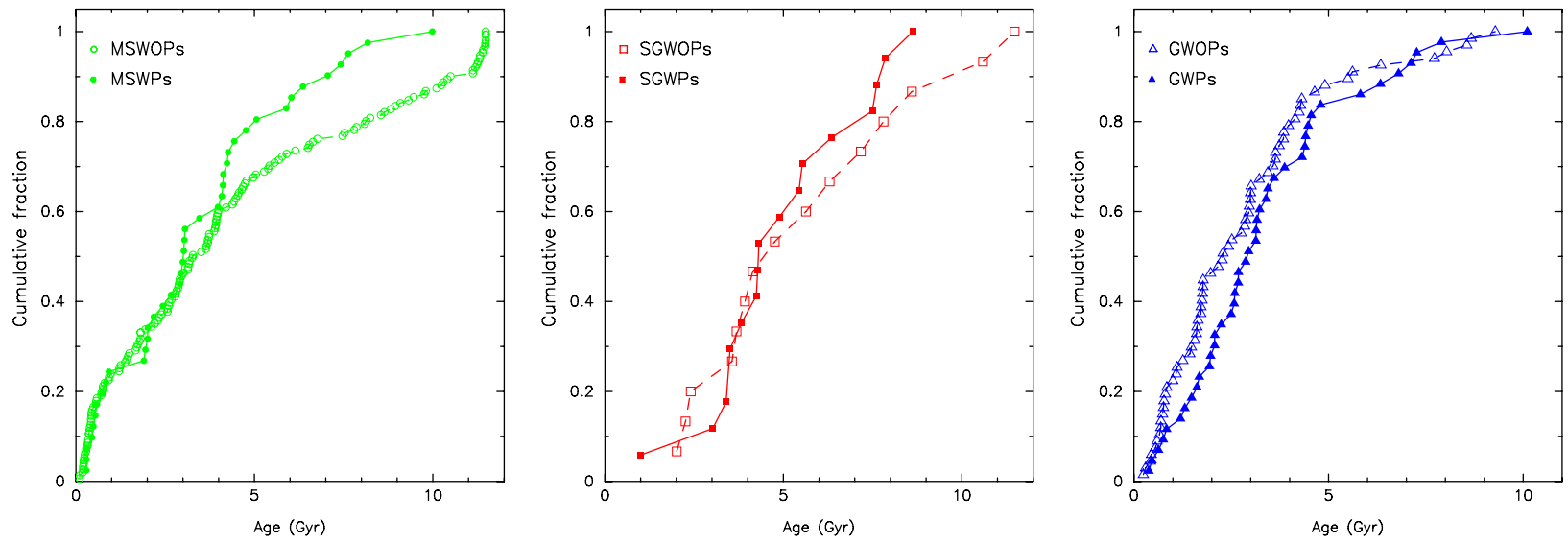

Fig. 6. Cumulative distribution function of the stellar age for MS stars (left), subgiants (middle), and giants (right).

Table 4. Results of the K-S tests performed in this work.

\begin{tabular}{lcccccc}
\hline \hline \multicolumn{7}{c}{ Stellar mass } \\
\hline Sample & $n_{\text {planets }}$ & $n_{\text {comparison }}$ & $n_{\text {eff }}$ & $D$ & $p$ & $H_{0}^{\ddagger}$ \\
\hline MS & 41 & 151 & 32 & 0.36 & $\sim 10^{-4}$ & 1 \\
Subgiants & 17 & 15 & 7 & 0.24 & 0.69 & 0 \\
Giants & 43 & 67 & 26 & 0.22 & 0.15 & 0 \\
\hline \multicolumn{7}{c}{ Stellar age } \\
\hline Sample & $n_{\text {planets }}$ & $n_{\text {comparison }}$ & $n_{\text {eff }}$ & $D$ & $p$ & $H_{0}^{\ddagger}$ \\
\hline MS & 41 & 151 & 32 & 0.18 & 0.24 & 0 \\
Subgiants & 17 & 15 & 7 & 0.17 & 0.95 & 0 \\
Giants & 43 & 67 & 26 & 0.22 & 0.15 & 0 \\
\hline
\end{tabular}

Notes. We consider a confidence level of $98 \%$ to reject the null hypothesis $H_{0}$ (both samples coming from the same underlying continuous distribution). $D$ is the maximum deviation between the empirical distribution function of Samples 1 and 2. The value $p$ corresponds to the estimated likelihood of the null hypothesis, a value that is known to be reasonably accurate for sample sizes for which $n_{\mathrm{eff}} \geq 4$. $^{(\dot{*})}(0)$ : accept null hypothesis; (1): reject null hypothesis.

\subsection{Mass segregation in giants and abundance trends}

As a whole, MA13 find that for giant stars there is no correlation between the presence of giant planets and the metallicity of the star. However, within the lack of correlation, a dependency on the stellar mass seems to be hidden. While the less massive giant stars with planets $\left(M_{\star} \leq 1.5 M_{\odot}\right)$ are not metal rich, the metallicity of the sample of massive $\left(M_{\star}>1.5 M_{\odot}\right)$ giant stars with planets is higher than that of a similar sample of stars without planets. It is therefore natural to ask whether there are no differences in the $\langle[\mathrm{X} / \mathrm{Fe}]\rangle-T_{\mathrm{C}}$ trends between stars that are more massive than $1.5 M_{\odot}$ and less massive giants.

Figure 7 shows the mean $\langle[\mathrm{X} / \mathrm{Fe}]\rangle-T_{\mathrm{C}}$ trend of GWPs and GWOPs for giants with $M_{\star} \leq 1.5 M_{\odot}$ and giants in the mass domain $M_{\star}>1.5 M_{\odot}$. The results of the corresponding linear fits are given in Table 6 . When considering all the elements, we find that the slopes are always negative with the only exception of the GWOP sample for $M_{\star}>1.5 M_{\odot}$. However, we note that, in this case, the slope is consistent with zero. If we only consider refractory elements for the more massive giants, we find that stars with and without planets show similar negative slopes. For giants in the mass domain $M_{\star} \leq 1.5 M_{\odot}$, GWPs show a slightly negative slope, whilst GWOPs show a slightly positive one. However, we note that both slopes, within their corresponding errors, are compatible with zero. We conclude that giant stars do not show differences in the $\langle[\mathrm{X} / \mathrm{Fe}]\rangle-T_{\mathrm{C}}$ trends between planet and non-planet hosts. This result holds independently of whether all giants are considered (Sect. 3.1) or if we separate the sample according to their stellar mass.

We note that giants in the mass domain $M_{\star} \leq 1.5 M_{\odot}$ show more positive $\langle[\mathrm{X} / \mathrm{Fe}]\rangle-T_{\mathrm{C}}^{\text {ref }}$ slopes than giants with $M_{\star}>$ $1.5 M_{\odot}$. This fact could, in principle, be explained by the anticorrelation between the stellar mass and the $T_{\mathrm{C}}^{\mathrm{ref}}$-slope seen in the previous subsection. Furthermore, a metallicity effect could be hidden since MA13 pointed out that giants in the mass domain 

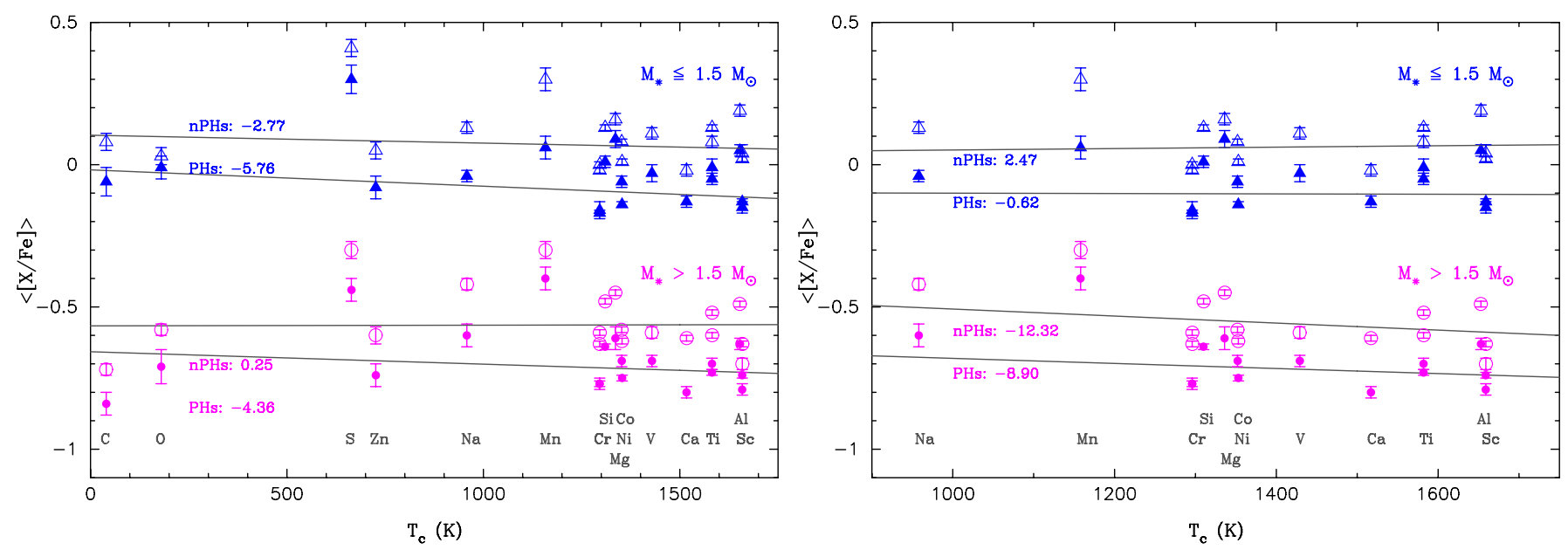

Fig. 7. $\langle[\mathrm{X} / \mathrm{Fe}]\rangle-T_{\mathrm{C}}$ trends for giant stars. GWPs with $M_{\star}>1.5 M_{\odot}$ are plotted with purple circles, GWPs less massive than $1.5 M_{\odot}$ in blue triangles. Filled symbols indicate planet hosts. Each planet host subsample is shown against its corresponding comparison subsample with an offset of -0.15 for the sake of clarity. The offset between the sample of giants with $M_{\star}>1.5 M_{\odot}$ and less massive giants is -0.75 . For guidance, the derived slopes are shown in the plots (units of $10^{-5} \mathrm{dex} / \mathrm{K}$ ). The left panel shows the $\langle[\mathrm{X} / \mathrm{Fe}]\rangle-T_{\mathrm{C}}$ trends when all elements (volatiles and refractories) are taken into account, whilst the one on the right shows the $\langle[\mathrm{X} / \mathrm{Fe}]\rangle-T_{\mathrm{C}}$ trends when only refractories are considered.

Table 5. Same as Table 2 but for a sample of 34 solar analogs $\left(T_{\text {eff }}=5777 \pm 200 \mathrm{~K}, \log g=4.44 \pm 0.20 \mathrm{dex},[\mathrm{Fe} / \mathrm{H}]=0.00 \pm 0.20 \mathrm{dex}\right)$.

\begin{tabular}{|c|c|c|c|c|c|c|c|c|}
\hline \multirow[b]{3}{*}{ Parameter } & \multicolumn{4}{|c|}{ All elements } & \multicolumn{4}{|c|}{ Only refractory } \\
\hline & \multicolumn{2}{|c|}{$\mathrm{MC}$} & \multicolumn{2}{|c|}{$\mathrm{CA}$} & \multicolumn{2}{|c|}{$\mathrm{MC}$} & \multicolumn{2}{|c|}{ CA } \\
\hline & SR & $\mathrm{ZS}$ & SR & $p$ & SR & $\mathrm{ZS}$ & SR & $p$ \\
\hline$[\mathrm{Fe} / \mathrm{H}]$ & $0.07 \pm 0.18$ & $0.18 \pm 0.44$ & 0.06 & 0.72 & $-0.38 \pm 0.15$ & $-0.96 \pm 0.43$ & -0.48 & $\sim 10^{-3}$ \\
\hline $\log g$ & $0.32 \pm 0.15$ & $0.79 \pm 0.40$ & 0.44 & 0.01 & .17 & $-0.05 \pm 0.42$ & 0.04 & 0.81 \\
\hline$M_{\star}$ & $0.13 \pm 0.17$ & $0.32 \pm 0.42$ & 0.13 & 0.47 & $-0.18 \pm 0.18$ & $-0.45 \pm 0.44$ & -0.22 & 0.20 \\
\hline Age & $-0.22 \pm 0.16$ & $-0.53 \pm 0.40$ & -0.29 & 0.10 & $0.08 \pm 0.18$ & $0.20 \pm 0.43$ & 0.06 & 0.72 \\
\hline$R_{\star}$ & $-0.16 \pm 0.18$ & $-0.38 \pm 0.43$ & -0.20 & 0.26 & $0.11 \pm 0.18$ & $0.26 \pm 0.43$ & 0.09 & 0.62 \\
\hline$d_{\text {galact }}$ & $-0.04 \pm 0.18$ & $-0.10 \pm 0.43$ & -0.05 & 0.77 & $-0.13 \pm 0.18$ & $-0.33 \pm 0.46$ & -0.15 & 0.39 \\
\hline
\end{tabular}

Table 6. Results of the $\langle[\mathrm{X} / \mathrm{Fe}]\rangle-T_{\mathrm{C}}$ linear fits for giant stars according to their masses.

\begin{tabular}{lcccc}
\hline \hline Sample & $\begin{array}{c}\text { All elements } \\
\text { Slope }\left(\times 10^{-5} \mathrm{dex} / \mathrm{K}\right)\end{array} \quad p \quad \begin{array}{c}\text { Only refractory } \\
\text { Slope }\left(\times 10^{-5} \mathrm{dex} / \mathrm{K}\right)\end{array}$ \\
\hline \multicolumn{6}{c}{$M_{\star}>1.5 M_{\odot}$} \\
\hline GWPs & $-4.36 \pm 1.58$ & 0.08 & $-8.90 \pm 2.42$ & 0.06 \\
GWOPs & $0.25 \pm 0.89$ & 0.05 & $-12.32 \pm 1.82$ & 0.05 \\
\hline \multicolumn{6}{c}{$M_{\star} \leq 1.5 M_{\odot}$} \\
\hline GWPs & $-5.76 \pm 1.58$ & 0.09 & $-0.62 \pm 2.35$ & 0.10 \\
GWOPs & $-2.77 \pm 1.20$ & 0.08 & $2.47 \pm 2.12$ & 0.07 \\
\hline
\end{tabular}

Notes. For each fit its probability of slope being by chance $(p)$ is also given.

$M_{\star} \leq 1.5 M_{\odot}$ show lower metallicities than giants with $M_{\star}>$ $1.5 M_{\odot}$. We note that the $T_{\mathrm{C}}^{\mathrm{ref}}$-slope has already been shown to depend on the stellar metallicity.

\section{Discussion}

In the previous section we have shown that stellar $T_{\mathrm{C}}$-slopes correlate with the stellar evolutionary parameters. The data suggest that there might be a different behaviours in the $\langle[\mathrm{Fe} / \mathrm{H}]\rangle-T_{\mathrm{C}}$ trends between planets and non-planet hosts for MS and subgiant stars. However, there seems to be no difference between planet and non-planet hosts among the sample of giants.
The finding that the MS non-planet hosts of our sample are less massive, and perhaps older than the planet hosts is, if significant, somehow surprising, and might hide some bias in the subsample selection. In fact, spectroscopic targets for planet searches are often deliberately chosen to be slow rotators and typically inactive, which should sample a population of MS stars older than the average population of the same spectral type. Recently, Bonfanti et al. (2015) have analysed whether exoplanet hosts are peculiar with respect to field stars that do not host planets in terms of age and found that both samples are homogeneous within the solar neighbourhood with a median age distribution of $4.8 \mathrm{Gyr}$, which is slightly older than the average thin-disc population. This seems to be at odds with our results, although it may be an effect of the sample selection. We note that our MS stars are younger on average (see Table 3 ). Further, the only difference found to be statistically significant between MSWPs and MSWOPs is in the stellar mass.

Haywood (2009) suggest that the observed correlation between the presence of gas-giant planets and enhanced stellar metallicity, observed in MS planet hosts, might be related to a possible inner-disc origin of these stars. The fact that stars with low-mass planets do not show the metal-rich signature does not necessary contradict this idea, although further investigations are needed to clarify this point. Radial mixing is a secular process, and its effect is known to increase with time; older stars migrate further and come from a region with significantly different abundances. On the other hand, age and mass are related quantities. MS non-planet hosts may show a different $[\mathrm{X} / \mathrm{Fe}]-T_{\mathrm{C}}$ trends 
(with respect to planet host) simply because these stars are slightly older and less massive, and possibly more contaminated by stars from the outer disc. Stars from the outer disc (at larger galactocentric distances) are expected to show lower metallicities (e.g. Lemasle et al. 2008, Fig. 5) and therefore larger [X/Fe] values for most elements (see Fig. A.1), which may explain their positive $T_{\mathrm{C}}^{\text {ref }}$ slopes. In this framework, the lack of a difference between GWPs and GWOPs is explained by the fact that these samples are younger and more massive than their MS counterparts, and therefore significantly less affected by radial mixing.

Along this line, hints of a correlation between the $T_{\mathrm{C}}$ slopes and the stellar age have already been reported (Adibekyan et al. 2014; Maldonado et al. 2015; Nissen 2015; Spina et al. 2016). Adibekyan et al. (2014) use the stellar mean galactocentric distance as a proxy of the stellar birthplace - finding tentative evidence of a correlation with the $T_{\mathrm{C}}$ slope. Such a correlation seems to also be present in our data (although when considering only refractory elements, see Table 2). Unfortunately, no values of $d_{\text {galact }}$ are available for most of our giant stars.

An alternative interpretation of the $\langle[\mathrm{Fe} / \mathrm{H}]\rangle-T_{\mathrm{C}}$ abundance patterns in planet hosts was given by Meléndez et al. (2009, hereafter ME09). ME09 report a deficit of refractory elements in the Sun with respect to other solar twins. ME09 conclude that the most likely explanation for this is related to the formation of planetary systems like our own, in particular to the formation of low-mass rocky planets.

In the analysis performed in Sect. 3 we have deliberately tried to exclude stars with known low-mass planets from the sample. Nevertheless, a difference between planet hosts and nonplanet hosts is still present in MS stars in the $T_{\mathrm{C}}^{\mathrm{ref}}$ analysis. This is in line with the results of MA15, where possible differences in abundance trends were found in stars with cool giant-planets, but not in stars with low-mass planets. Since the commonly accepted scenario of gas-giant planet formation requires the previous creation of a rocky core, the hypothesis that the atmospheres of planet hosts being contaminated by gas that was depleted in refractories, may still hold. The contamination of gas depleted in refractories owing to the planet formation process needs very accurate timing since the star needs to retain the protoplanetary disc long enough so that the planetary signatures are not cleared out by a deep convection zone on the star. Thus, as the star evolves off the MS to become a giant, this chemical fingerprint should be erased. In principle, at the base of the red giant branch phase most of the envelope should be fully convective. In this scenario subgiant stars are expected to show similar chemical fingerprint of planet formation as MS stars but giant stars should have it erased as planet hosts.

However, Fig. 4 (right) shows that the sample of stars that show hints of changing its chemical behaviour is the one without planets (from positive slopes for MSWOPs and SGWOPs to negative slopes for GWOPs). Thus, other explanations are required to explain this result. The presence of a galactic radial mixing is in agreement with the fact that we seem to be comparing two different populations of stars, with stars in the GWOP sample being more massive and younger than stars in the SGWOP and MSWOP samples.

It is important to bear in mind that the stars selected for planet searches around evolved stars are more massive than their MS counterparts (see, e.g. Niedzielski et al. 2016) and that it has been shown in MA13 and Reffert et al. (2015) that the planet occurrence rate does indeed seem to depend on both stellar mass and stellar metallicity. The different findings for lower and higher mass stars or MS and evolved systems does not appear to be simply a consequence of a polluted sample of planet hosts with non-planet-bearing stars. This explanation, put forward by Reffert et al. (2015), is what explains their sample of stars, but does not hold in our analysis of a larger sample (three times larger) even when we account for possible sample contamination. We find that we are indeed possibly dealing with different populations of stars, and we hope that improving sample statistics in the future will allow us to better clean-up the samples to reveal clues on planet formation processes under different conditions.

\section{Summary}

In this work, a detailed chemical analysis of a large sample of evolved stars (subgiants and red giants) with planets has been presented. Their chemical abundances has been compared to those of main-sequence stars.

No clear difference has been found in $\langle[\mathrm{X} / \mathrm{Fe}]\rangle-T_{\mathrm{C}}$ trends between planet and non-planet hosts when all elements are considered in the analysis. However, when the analysis is restricted to only refractory elements, planet and non-planet hosts may show different $T_{\mathrm{C}}$-slopes. This result holds for subgiant and giant stars, but not for giants.

The data suggest moderate but highly significant correlations between the $T_{\mathrm{C}}$-slopes and the stellar-evolutionary parameters, namely stellar mass and age. Less massive and older stars show more positive $T_{\mathrm{C}}^{\mathrm{ref}}$-slopes and more negative $T_{\mathrm{C}}^{\text {all }}$-slopes. As such, a hint of a difference in terms of mass and age seem to be present among our sample of MS stars, although this result should be further investigated, as it only seems to be statistically significant for the stellar mass. We also found that giants with masses $M_{\star} \leq 1.5 M_{\odot}$ show more positive $\langle[\mathrm{X} / \mathrm{Fe}]\rangle-T_{\mathrm{C}}^{\text {ref }}$ slopes than more massive giants, in agreement with their lower masses and metallicities.

Galactic radial mixing offers a suitable scenario for the observed trends. Giant stars are more massive and younger than their MS counterparts and, therefore, less contaminated by stars from the outer disc, leading to no chemical differences between planet and non-planet hosts. On the other hand, less massive and older stars in the MSWOP sample may account for different chemical trends between planets and non-planet hosts. Other scenarios invoking the formation of planets do not seem to be supported by our data.

Finally, we note that, while general trends between the $T_{\mathrm{C}}$ slopes and evolutionary parameters may be present, it does not exclude other processes, such as planetary formation, planet engulfment, or dust-gas segregation in protoplanetary discs that may affect the stellar photospheric abundance of refractory elements relative to volatiles (Gaidos 2015; Spina et al. 2016).

Acknowledgements. This research was supported by the Italian Ministry of Education, University, and Research through the "PREMIALE WOW 2013" research project under grant "Ricerca di pianeti intorno a stelle di piccola massa". E.V. acknowledges support from the "On the rocks" project funded by the Spanish Ministerio de Economía y Competitividad under grant AYA201455840-P. Carlos Eiroa is acknowledged for valuable discussions.

\section{References}

Adibekyan, V. Z., González Hernández, J. I., Delgado Mena, E., et al. 2014, A\&A, 564, L15

Allende Prieto, C., Lambert, D. L., \& Asplund, M. 2001, ApJ, 556, L63

Allende Prieto, C., Barklem, P. S., Lambert, D. L., \& Cunha, K. 2004, A\&A, 420, 183

Biazzo, K., Gratton, R., Desidera, S., et al. 2015, A\&A, 583, A135

Bonfanti, A., Ortolani, S., Piotto, G., \& Nascimbeni, V. 2015, A\&A, 575, A18

Bressan, A., Marigo, P., Girardi, L., et al. 2012, MNRAS, 427, 127 
Buchhave, L. A., \& Latham, D. W. 2015, ApJ, 808, 187

Buchhave, L. A., Latham, D. W., Johansen, A., et al. 2012, Nature, 486, 375 Casagrande, L., Schönrich, R., Asplund, M., et al. 2011, A\&A, 530, A138

Cassan, A., Kubas, D., Beaulieu, J.-P., et al. 2012, Nature, 481, 167

Curran, P. A. 2014, unpublished [arXiv: 1411.3816]

da Silva, L., Girardi, L., Pasquini, L., et al. 2006, A\&A, 458, 609

da Silva, R., Milone, A. C., \& Reddy, B. E. 2011, A\&A, 526, A71

da Silva, R., Milone, A. D. C., \& Rocha-Pinto, H. J. 2015, A\&A, 580, A24

Delgado Mena, E., Israelian, G., González Hernández, J. I., et al. 2010, ApJ, 725, 2349

Döllinger, M. P., Hatzes, A. P., Pasquini, L., Guenther, E. W., \& Hartmann, M. 2009, A\&A, 505, 1311

Fischer, D. A., \& Valenti, J. 2005, ApJ, 622, 1102

Frandsen, S., \& Lindberg, B. 1999, in Astrophysics with the NOT, eds.

H. Karttunen, \& V. Piirola, 71

Gaidos, E. 2015, ApJ, 804, 40

Ghezzi, L., \& Johnson, J. A. 2015, ApJ, 812, 96

Ghezzi, L., Cunha, K., Schuler, S. C., \& Smith, V. V. 2010a, ApJ, 725, 721

Ghezzi, L., Cunha, K., Smith, V. V., et al. 2010b, ApJ, 720, 1290

Girardi, L., Bressan, A., Bertelli, G., \& Chiosi, C. 2000, A\&AS, 141, 371

Gonzalez, G. 1997, MNRAS, 285, 403

Gonzalez, G. 2011, MNRAS, 416, L80

González Hernández, J. I., Israelian, G., Santos, N. C., et al. 2010, ApJ, 720, 1592

González Hernández, J. I., Delgado-Mena, E., Sousa, S. G., et al. 2013, A\&A, 552, A6

Gratton, R. G., Bonanno, G., Bruno, P., et al. 2001, Exp. Astron., 12, 107

Haywood, M. 2009, ApJ, 698, L1

Hekker, S., \& Meléndez, J. 2007, A\&A, 475, 1003

Howard, A. W., Sanchis-Ojeda, R., Marcy, G. W., et al. 2013, Nature, 503, 381

Jofré, E., Petrucci, R., Saffe, C., et al. 2015, A\&A, 574, A50

Johnson, J. A., Clanton, C., Howard, A. W., et al. 2011, ApJS, 197, 26

Kennedy, G. M., \& Kenyon, S. J. 2008, ApJ, 673, 502

Kjeldsen, H., \& Bedding, T. R. 1995, A\&A, 293, 87

Kurucz, R. 1993, ATLAS9 Stellar Atmosphere Programs and $2 \mathrm{~km} \mathrm{~s}^{-1}$ grid, Kurucz CD-ROM No. 13 (Cambridge, Mass.: Smithsonian Astrophysical Observatory)

Lemasle, B., François, P., Piersimoni, A., et al. 2008, A\&A, 490, 613

Lloyd, J. P. 2011, ApJ, 739, L49

Lloyd, J. P. 2013, ApJ, 774, L2

Lodders, K. 2003, ApJ, 591, 1220
Maldonado, J., Villaver, E., \& Eiroa, C. 2013, A\&A, 554, A84

Maldonado, J., Eiroa, C., Villaver, E., Montesinos, B., \& Mora, A. 2015, A\&A, 579, A20

Massarotti, A., Latham, D. W., Stefanik, R. P., \& Fogel, J. 2008, AJ, 135, 209

Mayor, M., Marmier, M., Lovis, C., et al. 2011, A\&A, submitted [arXiv: 1109.2497]

Meléndez, J., Asplund, M., Gustafsson, B., \& Yong, D. 2009, ApJ, 704, L66

Mortier, A., Santos, N. C., Sousa, S. G., et al. 2013, A\&A, 557, A70

Mustill, A. J., \& Villaver, E. 2012, ApJ, 761, 121

Niedzielski, A., Deka-Szymankiewicz, B., Adamczyk, M., et al. 2016, A\&A, 585, A73

Nissen, P. E. 2015, A\&A, 579, A52

Pasquini, L., Döllinger, M. P., Weiss, A., et al. 2007, A\&A, 473, 979

Pfeiffer, M. J., Frank, C., Baumueller, D., Fuhrmann, K., \& Gehren, T. 1998, A\&AS, 130, 381

Ramírez, I., Meléndez, J., \& Asplund, M. 2009, A\&A, 508, L17

Ramírez, I., Asplund, M., Baumann, P., Meléndez, J., \& Bensby, T. 2010, A\&A, 521, A33

Ramírez, I., Meléndez, J., \& Asplund, M. 2014, A\&A, 561, A7

Ramírez, I., Khanal, S., Aleo, P., et al. 2015, ApJ, 808, 13

Raskin, G., van Winckel, H., Hensberge, H., et al. 2011, A\&A, 526, A69

Reffert, S., Bergmann, C., Quirrenbach, A., Trifonov, T., \& Künstler, A. 2015, A\&A, 574, A116

Sadakane, K., Ohnishi, T., Ohkubo, M., \& Takeda, Y. 2005, PASJ, 57, 127

Santos, N. C., Israelian, G., \& Mayor, M. 2004, A\&A, 415, 1153

Schlaufman, K. C., \& Winn, J. N. 2013, ApJ, 772, 143

Schuler, S. C., Kim, J. H., Tinker, Jr., M. C., et al. 2005, ApJ, 632, L131

Sneden, C. A. 1973, Ph.D. Thesis, The University of Texas at Austin, USA

Sousa, S. G., Santos, N. C., Israelian, G., Mayor, M., \& Udry, S. 2011, A\&A, 533, A141

Spina, L., Meléndez, J., \& Ramírez, I. 2016, A\&A, 585, A152

Takeda, Y., Ohkubo, M., Sato, B., Kambe, E., \& Sadakane, K. 2005, PASJ, 57, 27

Takeda, Y., Sato, B., \& Murata, D. 2008, PASJ, 60, 781

Thiabaud, A., Marboeuf, U., Alibert, Y., Leya, I., \& Mezger, K. 2015, A\&A, 580, A30

Villaver, E., \& Livio, M. 2009, ApJ, 705, L81

Villaver, E., Livio, M., Mustill, A. J., \& Siess, L. 2014, ApJ, 794, 3

Wang, J., \& Fischer, D. A. 2015, AJ, 149, 14

Zieliński, P., Niedzielski, A., Wolszczan, A., Adamów, M., \& Nowak, G. 2012, A\&A, 547, A91 


\section{Appendix A: Abundance ratios as a function of stellar metallicity}
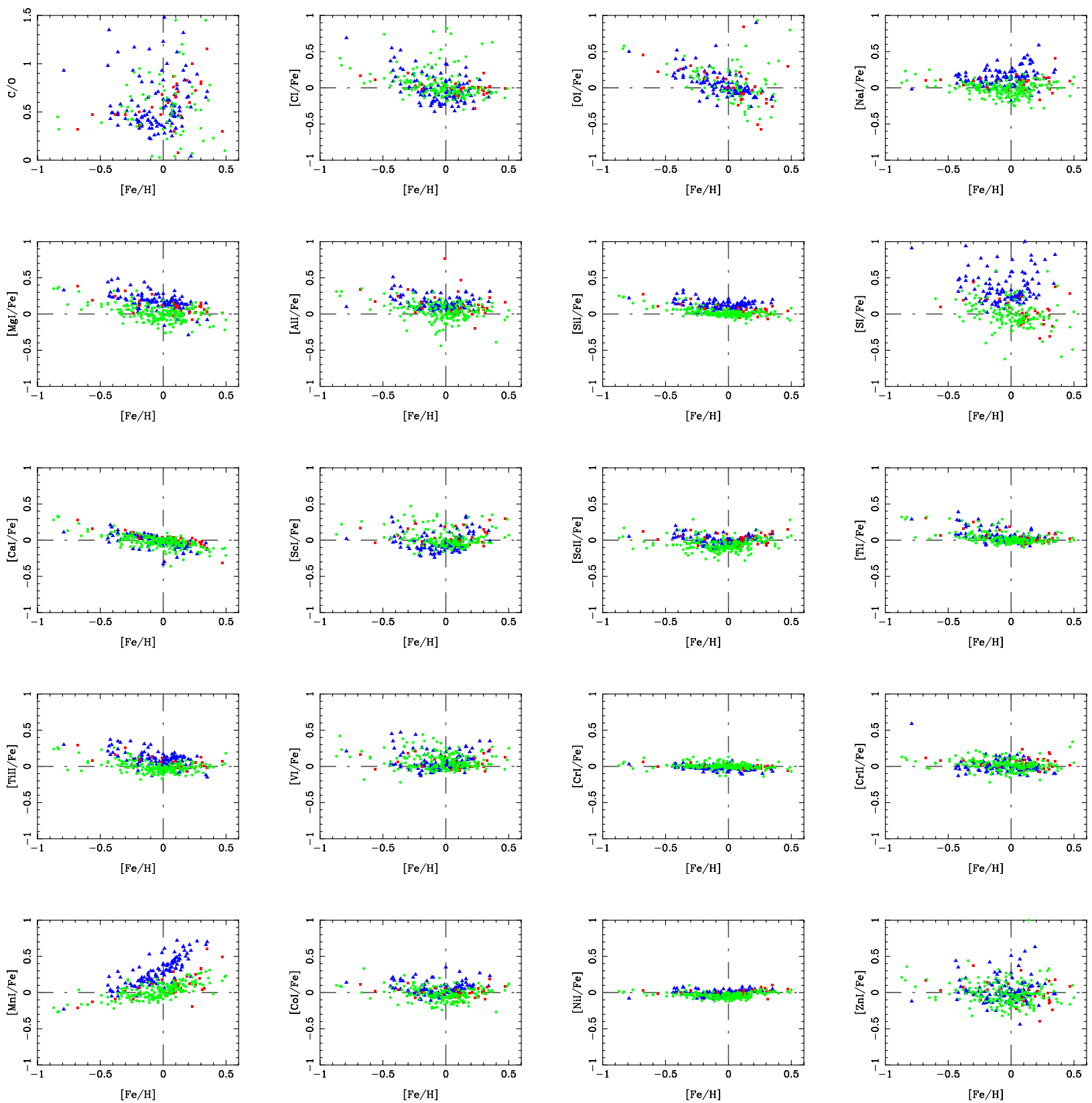

Fig. A.1. $\mathrm{C} / \mathrm{O}$ and chemical abundance ratios of $[\mathrm{X} / \mathrm{Fe}]$, as a function of the stellar metallicity. MS stars are plotted as green circles, subgiants as red squares, and giants as blue triangles.

\section{Appendix B: Description of data available at the CDS}

Results produced in the framework of this work are only available at the CDS.

Table B.1 lists all the stars analysed in this work. We note that the data for the majority of the main-sequence stars comes from MA15 and is not reproduced here. The table provides: HIP number (Col. 1); HD number (Col. 2); effective temperature in kelvin (Col. 3); logarithm of the surface gravity in $\mathrm{cms}^{-2}$ (Col. 4); microturbulent velocity in kms ${ }^{-1}$ (Col. 5); final metallicity in dex (Col. 6); spectrograph (Col. 7); stellar age in Gyr (Col. 8); stellar mass in solar units (Col. 9); and stellar radii in solar units (Col. 10). Each measured quantity is accompanied by its corresponding uncertainty.

Table B.2 gives the abundances of C I, O I, Na I, Mg I, Al I, Si I, S I, Ca I, Sc I, Sc II, Ti I, Ti II, V I (HFS taken into account), Cr I, Cr II Mn I, Co I (HFS taken into account), Ni I, and Zn I. They are expressed relative to the solar value, i.e. $[\mathrm{X} / \mathrm{H}]=\log \left(N_{\mathrm{X}} / N_{\mathrm{H}}\right)-\log \left(N_{\mathrm{X}} / N_{\mathrm{H}}\right)_{\odot}$. Note that the data for the majority of the main-sequence stars comes from MA15 and is not reproduced here. Abundances of carbon and oxygen of the stars from M15 were recomputed as described in Sect. 2. They are given in Table B.3. 